\title{
Die fossilen Araucaria-Zapfen der La Matilde Formation im versteinerten Wald von Cerro Cuadrado in Patagonien (Süd-Argentinien)
}

\author{
Veit Martin Dörken \& Hilke Steinecke
}

\section{Abstract}

Due to a volcanic eruption, the Araucaria dominated Jurassic forest of Cerro Cuadrado (Patagonia) got very well preserved. Particularly the seed cones of Araucaria mirabilis (Araucariaceae) belong to the most spectacular fossils of this formation. Their morphology is outlined.

\section{Zusammenfassung}

Durch einen Vulkanausbruch vor rund 160 Mio. Jahren wurde die damalige jurassische, von Araukarien dominierte Waldformation von Cerro Cuadrado (Patagonien) in einer einzigartigen Qualität konserviert. Zu den spektakulärsten Fossilien zählen hier zweifelsohne die Samenzapfen von Araucaria mirabilis (Araucariaceae), die nachfolgend vorgestellt werden.

\section{Einleitung}

Die Araukariengewächse (Araucariaceae) wie auch die Gattung Araucaria selbst, sind eine erdgeschichtlich sehr alte Gruppe von Gymnospermen (= Nacktsamern). Ihr Ursprung liegt wahrscheinlich im Trias-Zeitalter (vor rund 250-200 Mio. Jahren, Kunzmann 2007; Zonneveld 2012).

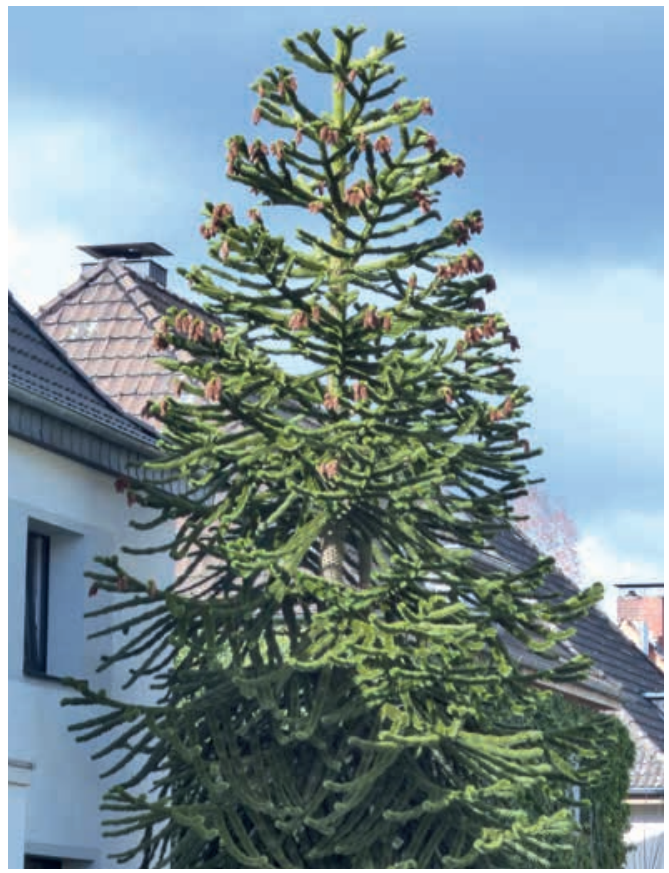

Abb. 1: Einst als kleines, langsam wachsendes Bäumchen in einem Vorgarten in Dortmund-Wambel gepflanzt und mittlerweile haushoch: Männliches Exemplar von Araucaria araucana. (Foto: G. Steinecke)
Die Familie hatte früher ein wesentlich weiteres Verbreitungsareal, als das heutige Artenspektrum dies noch vermuten lässt. So waren die Araucariaceae im Erdmittelalter (Mesozoikum, vor rund 250 bis 100 Mio. Jahren) auch auf der Nordhemisphäre verbreitet, während sich die heutige Verbreitung ausschließlich auf die Südhemisphäre beschränkt

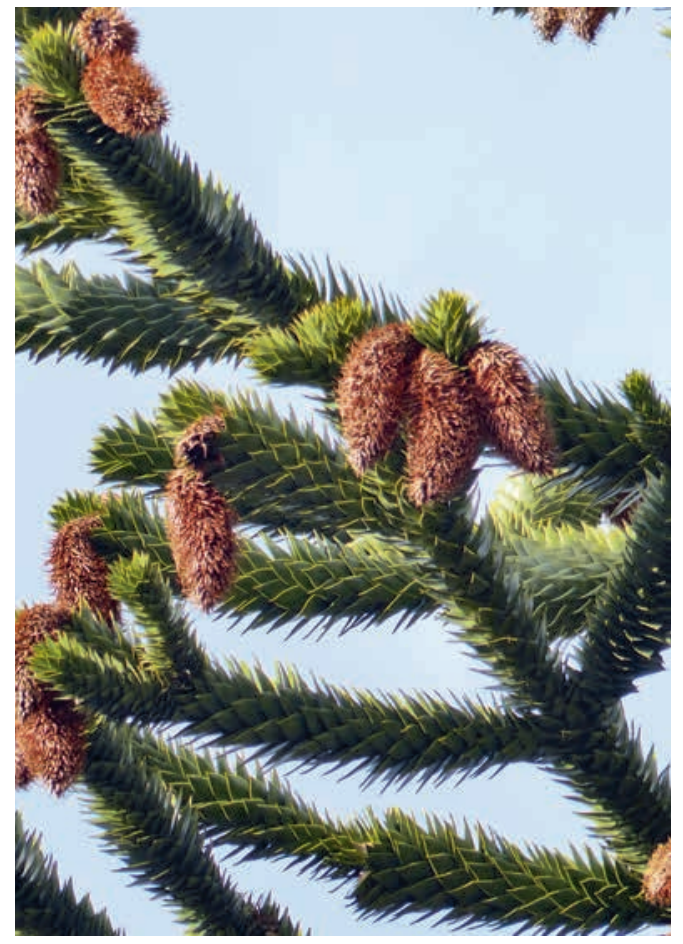

Abb. 2: Zweig von Araucaria araucana mit Pollenzapfen. (Foto: G. Steinecke) 


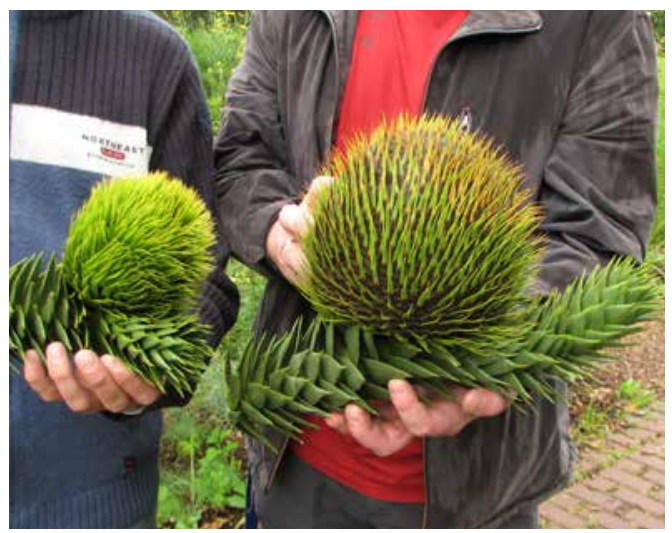

Abb. 3: Weibliches Exemplar mit Samenzapfen im Botanischen Garten in Düsseldorf. (Foto: V. M. Dörken).

(Kräusel 1922, Kampmann 1983, Stockey 1975, 1980, 1982, 1994; Stockey et al. 1994, Kunzmann 2007, Taylor et al 2009, Shaolin 2008, Nimsch 2011). Da es sich bei den Araucariaceae durchweg um Holzgewächse handelt, sind diese, vor allem Arten der Gattung Araucaria, auch als Fossilien vielfach erhalten. Fossilien von Araucaria carolae sind auch aus Deutschland bekannt (Kunzmann 2007). Meist handelt es sich hierbei größtenteils „nur“ um fossile Hölzer oder beblätterte Triebe. Seltener sind auch reproduktive Strukturen der Gattung Araucaria fossil dokumentiert, wobei Funde der weiblichen Samenzapfen im Vergleich zu den männlichen Pollenzapfen wesentlich häufiger vorkommen, insofern man von häufig in diesem Zusammenhang überhaupt sprechen darf. Die wohl eindrucksvollsten und mit am besten erhaltenen Samenzapfen stammen aus der La Matilde Formation von Cerro Cuadrado in Patagonien (Süd-Argentinien). Hier war Araucaria mirabilis eine der dominierenden Baumarten. Durch einen Vulkanausbruch im mittleren Jura, vor rund 160 Millionen Jahren, wurden die dortigen Araukarienwälder mit einer mächtigen Schicht aus Vulkanasche bedeckt. Durch diesen Vulkanausbruch ist der ehemalige Wald von Cerro Cuadrado in einem spektakulären Erhaltungszustand konserviert worden (Dernbach 1996). Außer Ästen und Stammstücken werden auch Samenzapfen und sogar Sämlinge in exzellentem Erhaltungszustand gefunden. An diesen rund 160 Millionen Jahre alten Fossilien können sogar noch die Gewebestrukturen im Detail studiert werden. Durch die Bedeckung mit Vulkanasche kam es zur Verkieselung der Pflanzenteile (TAYlor et al. 2009).

Männliche Pollenzapfen wurden im versteinerten Wald von Cerro Cuadrado so gut wie nicht nachgewiesen (Dernbach 1992, Taylor et al. 2009). Die wenigen männlichen Pollenzapfen wurden dann auch nicht Araucaria mirabilis zugeordnet, sie stammen möglicherweise von Pararaucaria patagonica, einer anderen Araukarie mit deutlich kleineren Samenzapfen (Dernbach 1992).

Je nach Autor wird das Fehlen der Pollenzapfen dahingehend gedeutet, dass die Bedeckung des Waldes mit Vulkanasche zu einem Zeitpunkt stattfand, bevor sich Pollenzapfen entwickelt hatten. Sicherlich ist aber auch von Bedeutung, dass Pollenzapfen wesentlich weniger stabil sind als Samenzapfen und sich deshalb generell schlechter in Form von Fossilien erhalten. Die Embryonen,

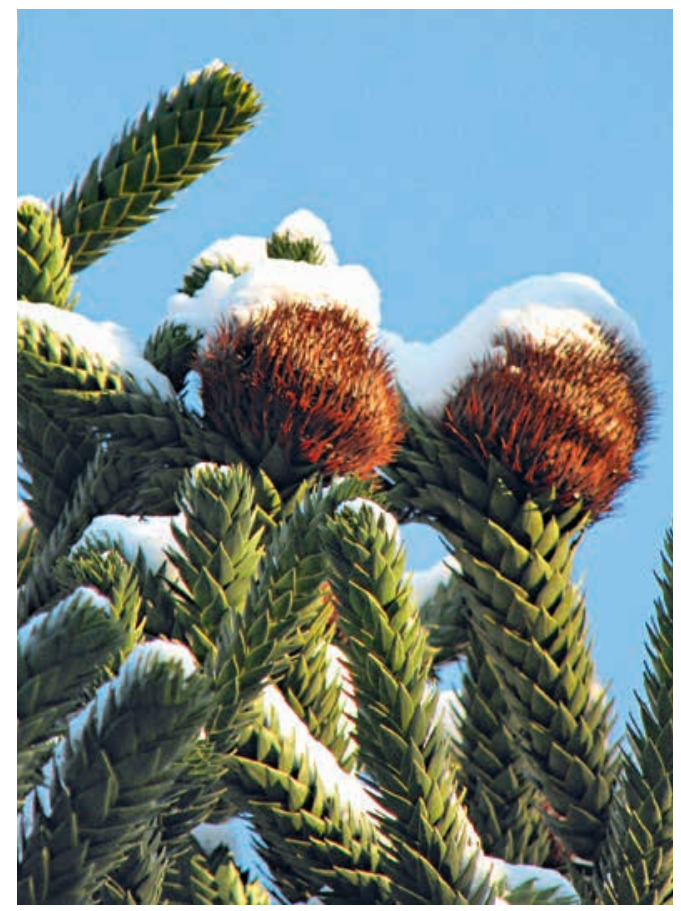

Abb. 4: Zweige von Araucaria araucana mit Samenzapfen aus dem Botanischen Garten Düsseldorf.

(Foto: V. M. DöRKEN) 


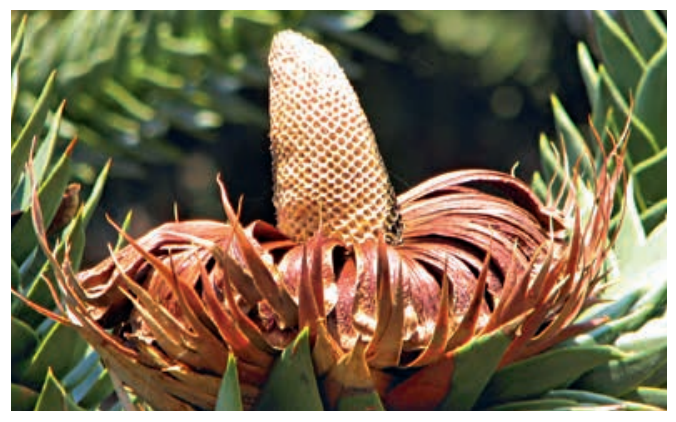

Abb. 5: Am Baum zerfallender Zapfen von Araucaria araucana. (Foto: V. M. DörkeN)

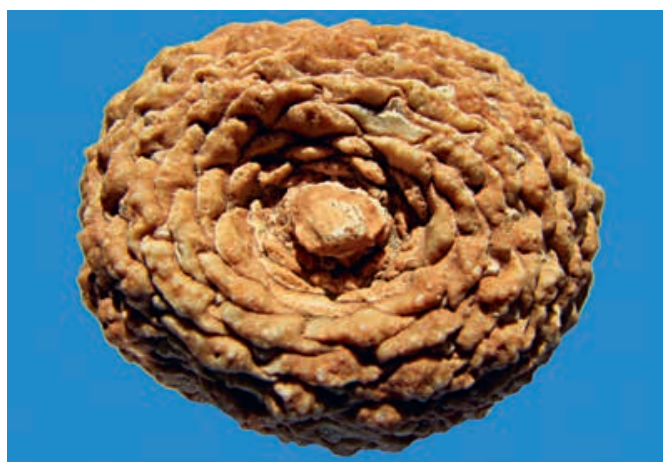

Abb. 6: Araucaria mirabilis, fossiler Zapfen in der Aufsicht, der kurze Zapfenstiel ist deutlich zu erkennen. (Foto: V. M. Dörken).

die trotz Abwesenheit von Pollen in den Samen ausgebildet sind, könnten sich daher parthenokarp entwickelt haben (TAYLOR et al. 2009).

Nachfolgend soll der Bau der Samenzapfen von Araucaria mirabilis genauer vorgestellt warden. Die Erstbeschreibung des versteinerten Waldes von Cerro Cuadrado in Patagonien erfolgte durch Wieland (1929). Vergleicht man den Bau der Zapfen der fossil nur aus Patagonien bekannten Art A. mirabilis mit denen der heutigen Araukarien, so zeigen sich große Übereinstimmungen zur rezenten Art A. bidwillii aus Queensland (Australien) (Stockey 1975, 1982, STockey \& TAYlor 1978, Dernbach 1992, Taylor et al. 2009). Die Samenzapfen beider Arten unterscheiden sich prinzipiell nur in der Zapfengröße voneinander, wobei die Zapfen von $A$. mirabilis deutlich kleiner bleiben als die von A. bidwillii. Aufgrund der strukturellen Ähnlichkeiten im Zapfenbau ist es wahrscheinlich, dass innerhalb der Araucariaceae die fossile

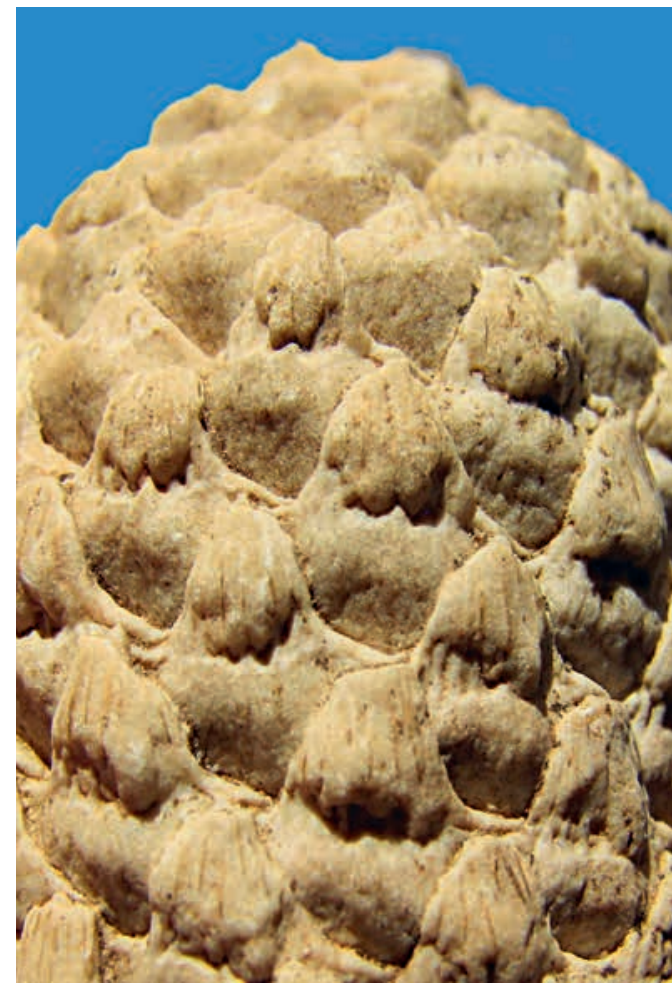

Abb. 7: Araucaria mirabilis, Details der Zapfenschuppen, die kleinen aufgesetzten Spitzen wurden im Regelfall frühzeitig abgeworfen und sind daher meist nicht erhalten geblieben.

Art A. mirabilis zur Sektion Bunya gestellt werden kann (Stockey 1975, 1982, Stockey \& TAYlor 1978, TAYlor et al. 2009). Diese Sektion umfasst aktuell nur die einzige rezente Art Araucaria bidwillii (FARJON 2010, Zonneveld 2012). Bemerkenswert ist, dass $A$. bidwillii heute nicht mehr auf dem südamerikanischen Kontinent vertreten ist. Die beiden heute in Südamerika heimischen Arten $A$. araucana (Andentanne) sowie $A$. angustifolia (Schmalblättrige Araukarie) gehören einer anderen Sektion (Araucaria) an (ECKENWALdER 2009, FARJon 2010). Fossil sind weitere Araukarien-Zapfen der Sektion Bunya bekannt, sie wurden in Europa, Südamerika sowie Indien gefunden. Dies lässt vermuten, dass die Sektion Bunya früher ein sehr großes Verbreitungsgebiet hatte (STOCKey 1982).

Da die attraktiven verkieselten Araukarienzapfen aus Cerro Cuadrado zu den besterhaltensten pflanzlichen Fossilien überhaupt gehören, sind 


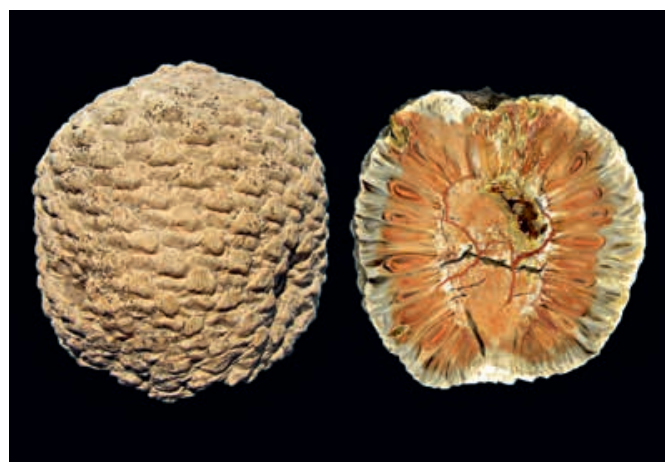

Abb. 8: Araucaria mirabilis, fossiler Zapfen in Seitenansicht und im Längsschnitt. (Foto: V. M. DöRkeN)

sie bei Sammlern extrem beliebt. Hinzu kommt, dass jeder Zapfen etwas unterschiedlich gefärbt ist durch Einlagerung z. B. von Chalcedon, Jaspis oder Carneol (Dernbach 1992). Zum Schutz des versteinerten Waldes wurde bereits 1954 das Schutzgebiet Bosques Petrificados eingerichtet, wobei das Sammeln der Zapfen und deren Export noch nicht verboten waren. Früher wurden die Zapfen (ganz oder halbiert und poliert) noch auf Fossilienbörsen zum Kauf angeboten. Seit einigen Jahren stehen die Fundstellen in Patagonien unter Grabungsschutz, so dass keine Neuaufsammlungen mehr auf den Markt kommen. Dementsprechend sind die Zapfen auf Börsen sehr selten geworden und stammen nur noch aus Auflösungen alter Sammlungen. Ihr Preis ist deutlich gestiegen. Abhängig von Größe und Qualität muss man mittlerweile mehrere hundert Euro für einen längs geschnittenen und polierten Zapfen ausgeben.

\section{Die Samenzapfen, gut erhaltene „Kunstwerke der Natur“}

Durch den jurassischen Vulkanausbruch vor rund 160 Millionen Jahren wurde die damalige Waldformation plötzlich von vulkanischen Sedimenten überdeckt und daher ist es nicht verwunderlich, dass die Samenzapfen aller Individuen mehr oder weniger in demselben Entwicklungsstadium konserviert wurden und selbst Details erhalten geblieben sind. Bei den Samenzapfen handelt es sich um fortgeschrittene Entwicklungsstadien, die deutlich hinter der Bestäubungs- und Befruchtungsphase liegen. Es gibt so gut wie keine Zapfen ohne Em-

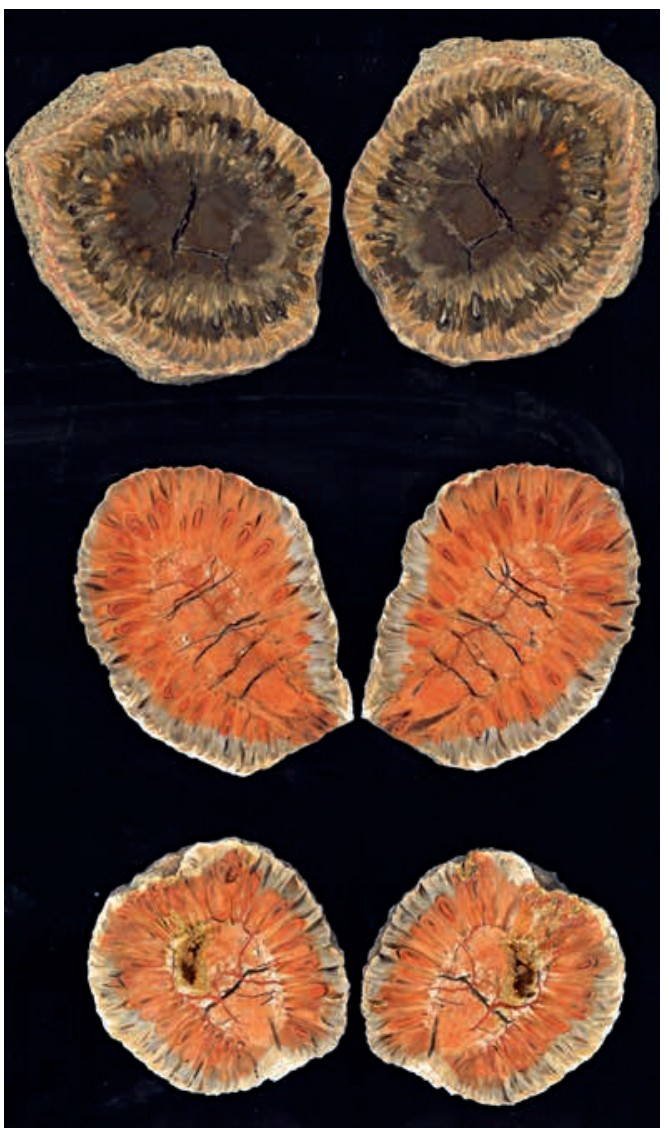

Abb. 9: Araucaria mirabilis, fossile Zapfen im Längsschnitt. (Foto: V. M. DöRKEN)

bryonen in den Samen. Die Samenzapfen haben eine rundliche bis elliptisch-eiförmige Gestalt und sind 3-8 $(-12) \mathrm{cm}$ lang und 3-8 $(-10) \mathrm{cm}$ breit. Der blattlose Stiel ist bei den meisten Zapfen recht kurz oder nur schlecht erhalten und übersteigt meist kaum eine Länge von $1,5 \mathrm{~cm}$.

Die Samenzapfen bauen sich aus zahlreichen Deck-/Samenschuppen-Komplexen auf, bei denen die Deckschuppe das Tragblatt für eine fruchtbare Seitenachse, die Samenschuppe, darstellt. Die Zapfenspindel ist im Vergleich zum Zapfendurchmesser recht breit und weist ein konzentrisches Leitbündelsystem mit Innenxylem und Außenphloem auf. Im Inneren ist ein mächtiger Markbereich aus großlumigen, dünnwandigen, parenchymatischen Zellen ausgebildet. 


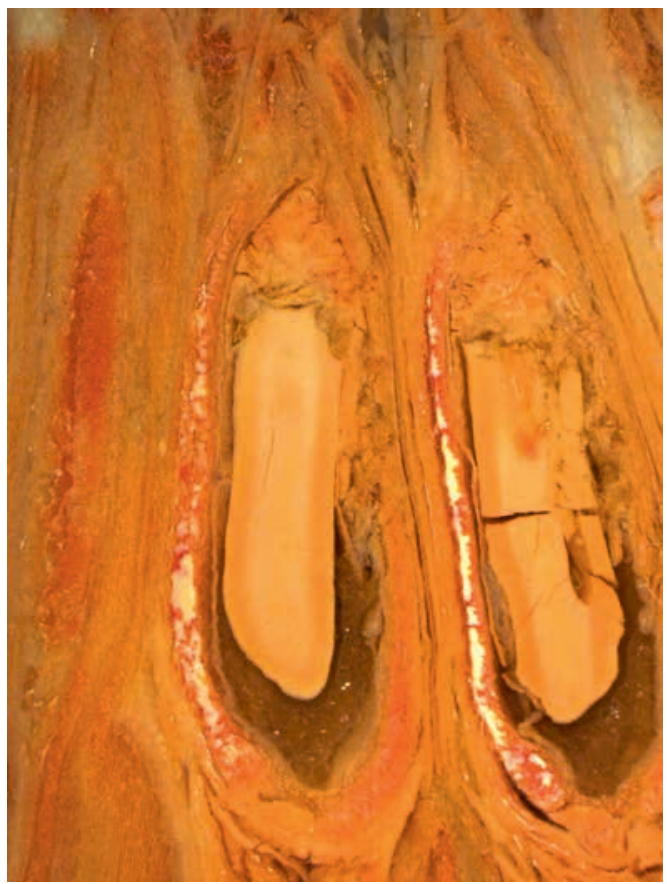

Abb. 10: Araucaria mirabilis, Samenanlagen mit bereits deutlich entwickeltem Embryo. (Foto: V. M. Dörken)

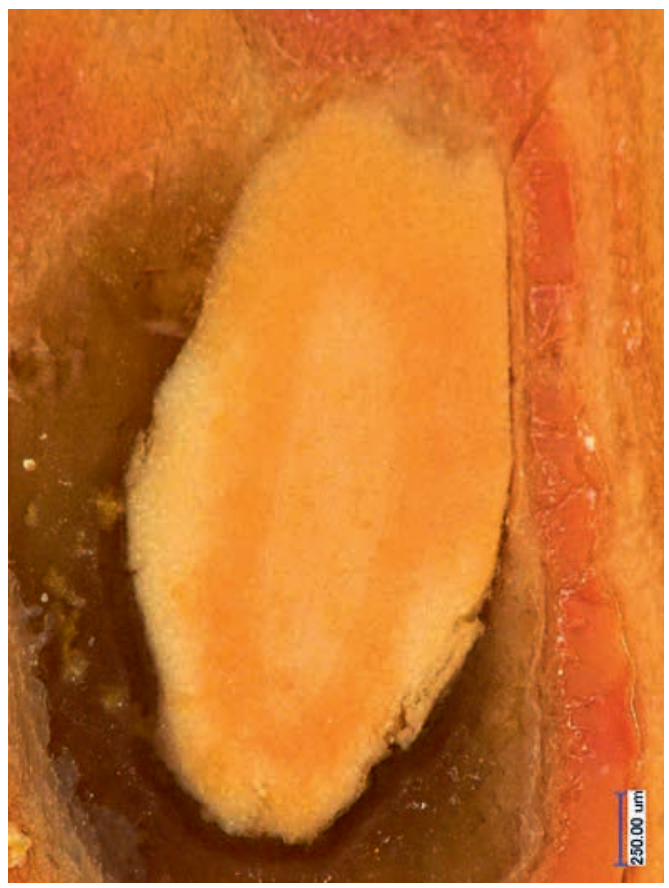

Abb. 11: Araucaria mirabilis, Detail eines Embryos mit zwei Keimblättern, Embryo von Nährgewebe (Endosperm) umschlossen. (Foto: V. M. Dörken)

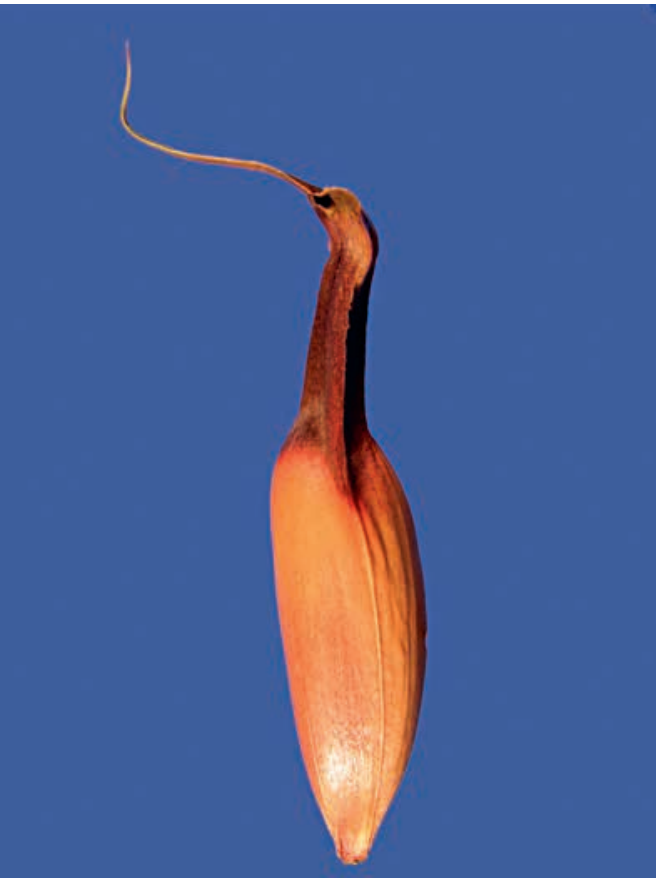

Abb. 12: Reifer rezenter Same von Araucaria araucana. (Foto: V. M. DöRKeN)

Deck- und Samenschuppe sind bei $A$. mirabilis bis etwa zur Hälfte miteinander verwachsen. Der freie distale Bereich der Samenschuppe wird häufig als Ligula bezeichnet, der freie Bereich zwischen Deck- und Samenschuppe als ligulärer Sulkus. Die Samenschuppe ist mit 1-3,5 (4) cm Länge und $0,5-1,5(-2) \mathrm{cm}$ Breite deutlich größer als die Deckschuppe und überragt diese deutlich. Die Leitbündelversorgung des Deck-/Samenschuppenkomplexes ist doppelt. Sowohl die Deck- als auch die Samenschuppe verfügen über einen jeweils getrennten Leitbündelanschluss mit Leitbündeln, die getrennt voneinander aus dem konzentrischen Leitbündelstrang der Zapfenspindel heraus in die Zapfenschuppen einziehen. Dabei werden die Leitbündel unterhalb stets von einem Harzkanal flankiert. Sowohl die Deck- als auch die Samenschuppe verholzen mit zunehmender Samenreife extrem stark.

Bei Araucaria mirabilis wie auch bei allen anderen heutigen Araukarien-Arten wird je Samenschuppe nur eine einzige Samenanlage ausgebildet. Die Mikropyle der Samenanlagen zeigt dabei zur 


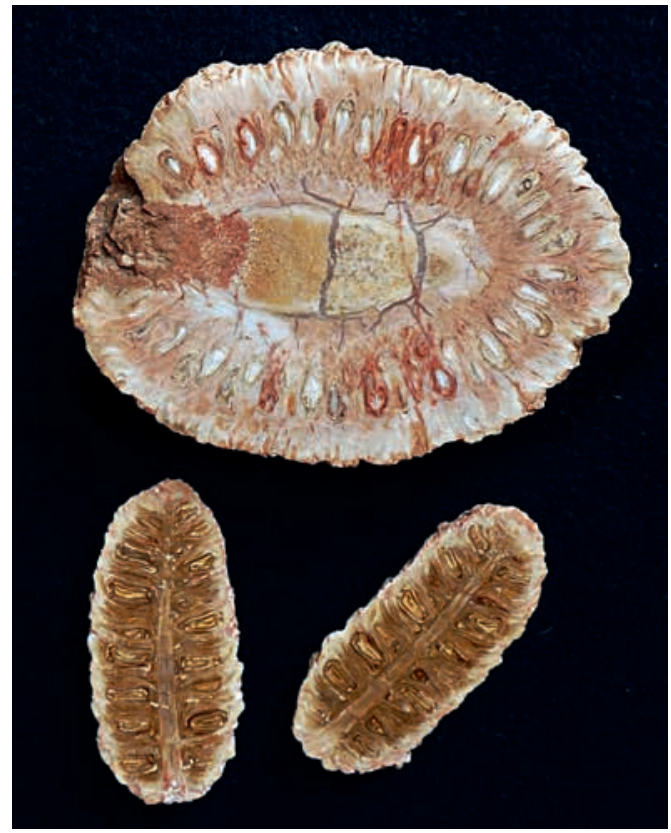

Abb. 13: Längs geschnittener fossiler Zapfen von Pararaucaria spec. neben einem verkieselten Zapfen von Araucaria mirabilis zum Vergleich. Die Zapfen von Parauracaria sind kleiner und schmaler als diejenigen von Araucaria mirabilis, ansonsten aber ähnlich aufgebaut und ebenso gut erhalten. (Foto: H. STeinecke)

Zapfenspindel hin. Das Integument der Samenanlage ist dreischichtig, die spätere Samenschale besteht aus einer fleischigen äußeren Sarkotesta, einer stark verholzten Zwischenschicht (Sklerotesta) und einer inneren dünnen Endotesta, die jedoch nur gelegentlich erkennbar ist. Das Makrosporangium (der so genannte Nucellus), in dem das Makroprothallium mit den Archegonien ausgebildet wird, ist größtenteils nicht mit dem umgebenden Integument verwachsen. Lediglich der der Mikropyle abgewandte Bereich des Nucellus ist mit der innersten Integumentschicht verwachsen.

Die reifen, ungeflügelten Samen erreichen eine Länge von $0,5-1(1,5) \mathrm{cm}$ und eine Breite von $0,2-0,5(-0,8) \mathrm{cm}$. Es scheint, als hätten sich die Samen, wie dies heute auch noch bei $A$. bidwillii der Fall ist, zur Reife von der Samenschuppe abgelöst und wurden separat von diesen ausgebreitet. Die junge Embryonen in den Samen haben eine Länge von rund 2-3 (-5) mm und einen Durch-

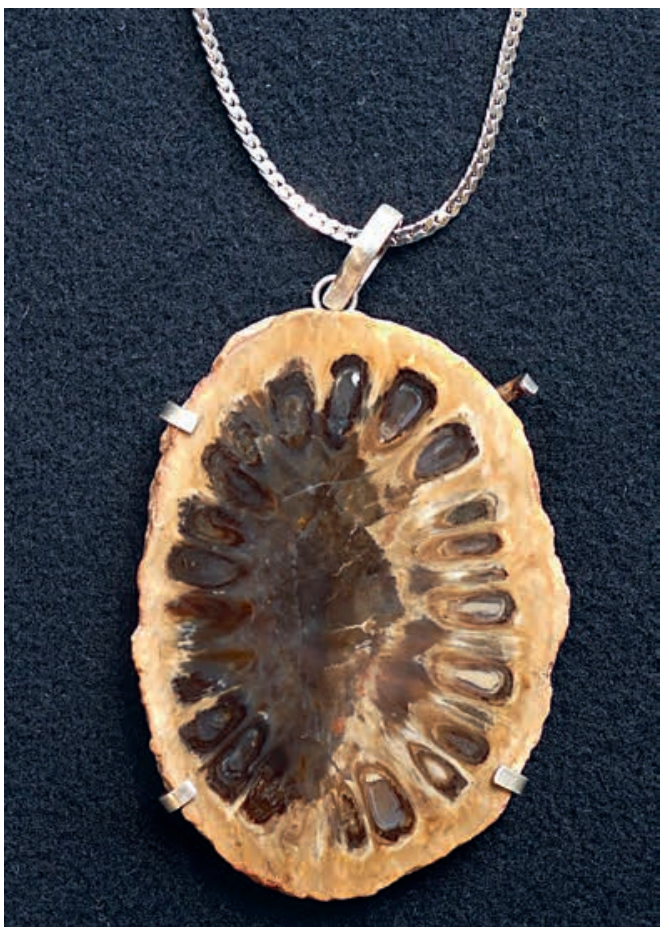

Abb. 14: Schmuck aus einer längs geschnittenen fossilen Zapfenscheibe von Araucaria mirabilis.

(Foto: H. Steinecke)

messer von 0,8-1,2 mm. Die Embryonen sind dabei im Nährgewebe meist parallel zur Samenschuppe hin ausgerichtet. Seltener sind sie senkrecht zur Zapfenschuppe hin orientiert (STOCKEY 1975). Die Embryonen bilden zwei Keimblätter wie auch bei $A$. bidwilii (FArJon 2010). Der Erhaltungsgrad der meisten Embryonen ist so exzellent, dass auch noch andere Merkmale wie z. B. die Sprossspitze, die Wurzelanlagen und die Leitbahnen deutlich erkennbar sind. In selteneren Fällen kann sogar auch der Suspensor, der Träger der Embryonen, erkannt werden.

Araukarien, genauer gesagt die Andentanne (Araucaria araucana), wurden vor einigen Jahrzehnten zu Modepflanzen von Vorgärten. Mittlerweile sind einige von ihnen groß geworden und entwickeln regelmäßig je nach Geschlecht Pollenbzw. Samenzapfen. Beim Anblick dieser bizarren Exoten sollte man sich immer wieder vergegenwärtigen, dass es sich um lebende Fossilien handelt und dass es Araukarien schon gab, als die Saurier 


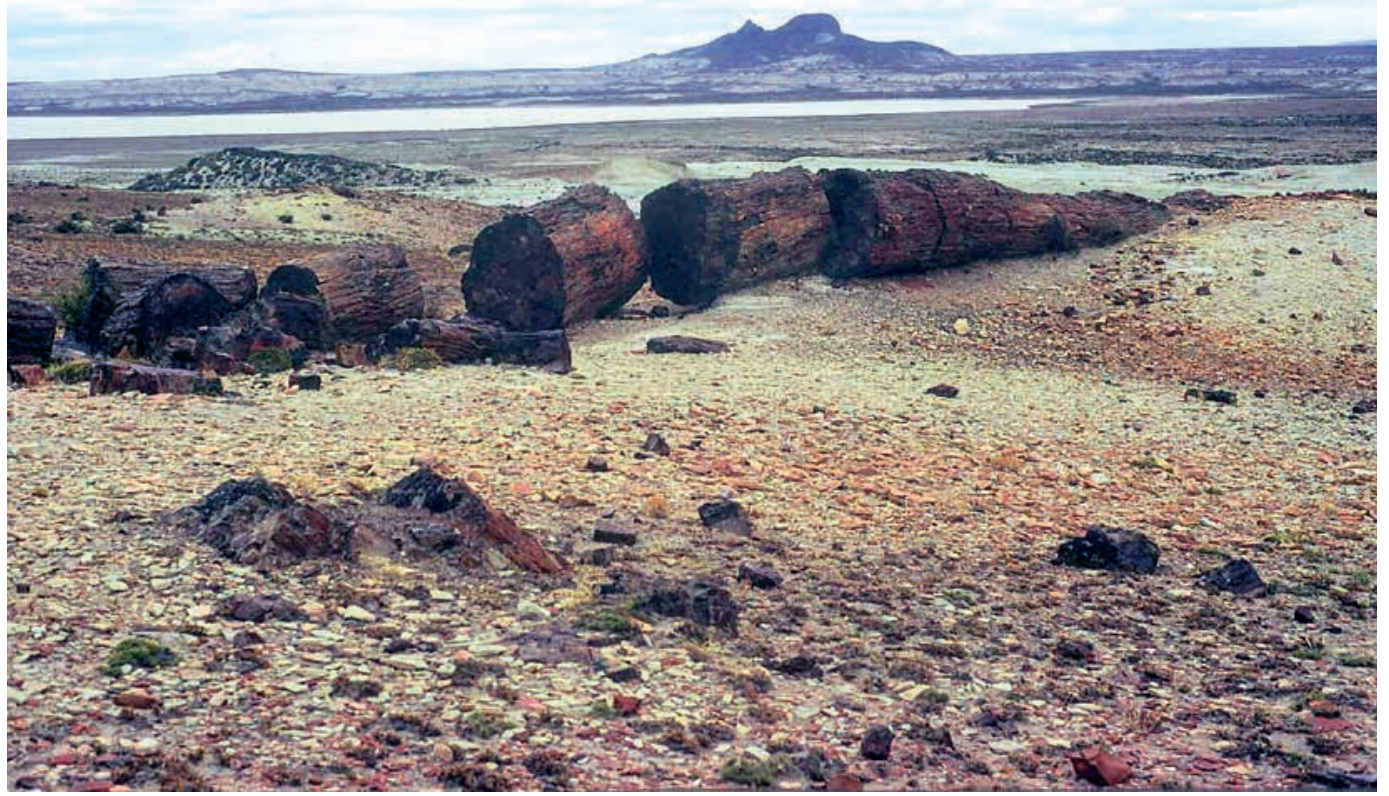

Abb. 15: Argentinien, Nationalpark Bosques Petrificados, $30 \mathrm{~m}$ langer gebrochener fossiler Stamm von Araucaria mirabilis. (Foto: H. Nimsch)

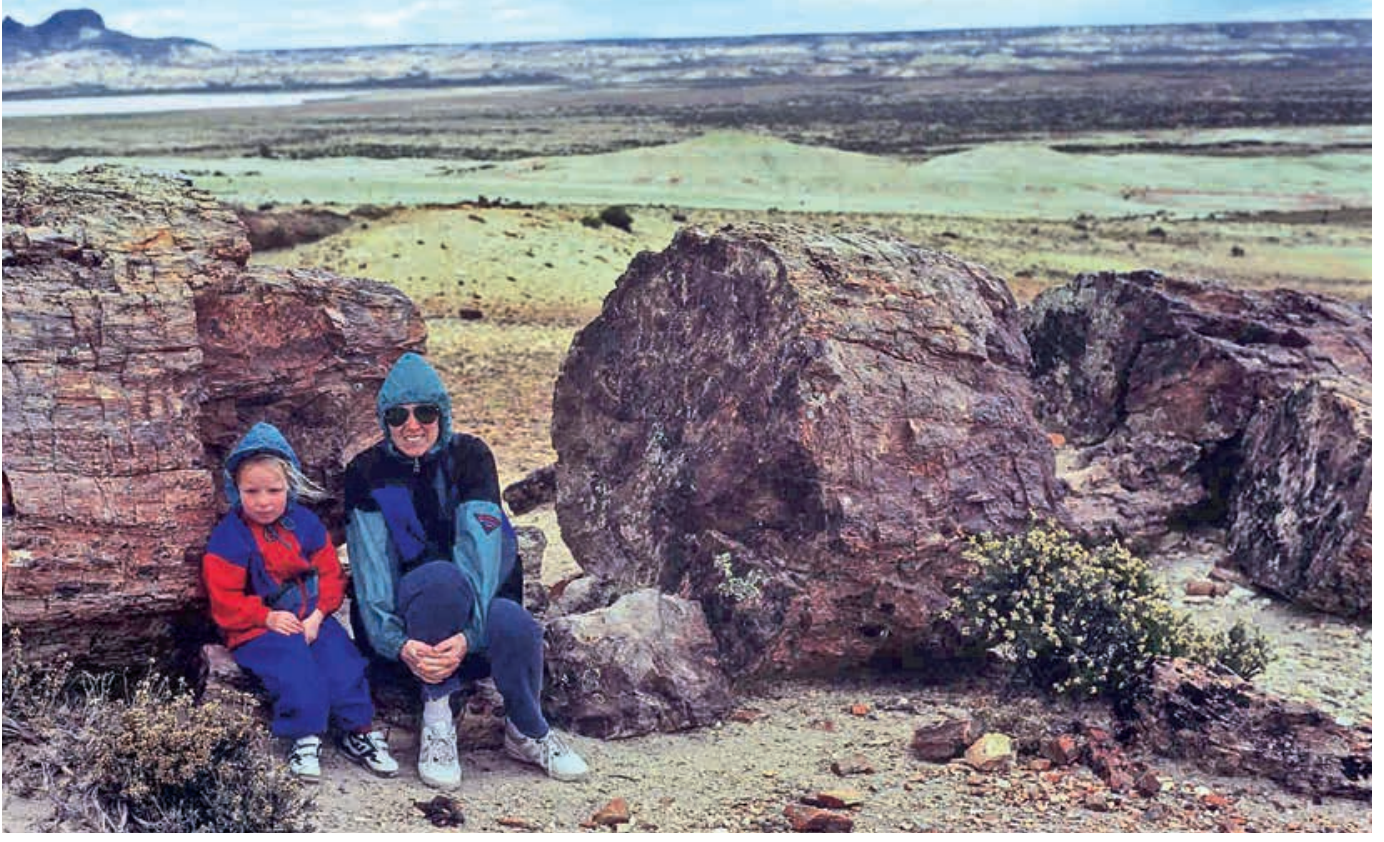

Abb. 16: Argentinien, Nationalpark Bosques Petrificados, Besucherinnen zum Größenvergleich neben einem fossilen Stamm von Araucaria mirabilis. (Foto: H. Niмsch) 


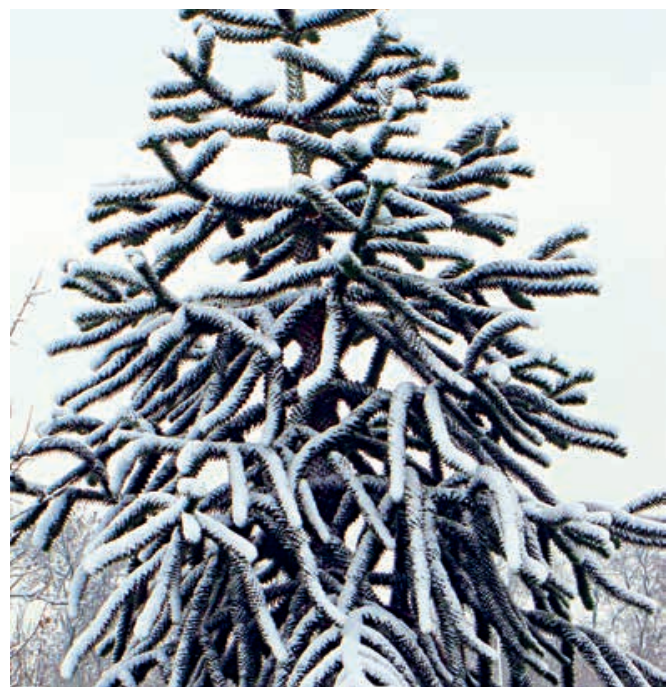

Abb. 17: Ob die versteinerten Zapfen oder die lebenden Bäume: Araukarien sind äußerst attraktiv. Besonders bizarr wirken die Bäume im Schnee, hier ein Exemplar in Dortmund. (Foto: G. Steinecke)

noch lebten. Wer die Gelegenheit hat, originale fossile Araukarienzapfen zu bewundern, sollte sie durchaus einmal mit rezenten Araukarienzapfen vergleichen. Die fossilen Araukarienzapfen aus Patagonien sind übrigens nicht nur eine Zierde in Fossiliensammlungen. Quer- und Längsschnitte findet man gelegentlich auch in zu Schmuck verarbeiteter Form.

\section{Dank}

Ganz herzlicher Dank gilt Hubertus Nimsch (St. Ulrich, Bollschweil) für die Bereitstellung von Fotos

\section{Literatur}

Dernbach, D. \& U. 1992 : Araucaria. Die versteinerten Araukarien vom Cerro Cuadrado, Argentinien. - Lorsch.

Dernbach, D. \& U. 1996 : Versteinerte Wälder. Die 31 schönsten versteinerten Wälder. - Lorsch

Eckenwalder, J. E. (2009): Conifers of the world. - Portland.

Farjon, A. 2010: A handbook of the world's conifers. - Leiden.

Kampmann, H. 1983: Mikrofossilien, Hölzer, Zapfen und Pflanzenreste aus der unterkretazischen Sauriergrube bei Brilon-Nehden. - Geol. Paläont. Westf. 1: 1-146.
KRÄUsEL, R. 1922: Beiträge zur Kenntnis der Kreideflora: über einige Kreidepflanzen von Swalmen (Niederlande). - Meded. Rijks Geol. Dienst Ser. A, No. 2: 1-40.

Kunzmann L. 2007: Neue Untersuchungen zu Araucaria Jussieu aus der europäischen Kreide. - Palaeontographica B, 276: 97-131.

Nimsch, H. 2011: Araucaria, alle Arten der Gattung in Bild und Text. - Remagen.

Shaolin, Z., Lidong, Z., Wu, Z \& Yajun, Y. 2008: A new female cone, Araucaria beipoiaoensis sp. nov. from the middle jurassic tiaojishan formation, Beipiao, Western Liaoning, China and its evolutionary significance. - Acta Geologica Sinica-English Edition 82: 266-282.

Stockey, R. A., Nishida, H. \& Nishida, M. 1994: Upper cretaceous Araucarian cones from Hokkaido and Saghalien: Araucaria nipponensis sp. nov. - Int. J. Plant Sci. 155: 806-815.

Stockey, R. A. \& TAYlor, T. N. 1978: On the structure and evolutionary relationships of the Cerro Cuadrado fossil conifer seedlings. - Bot. J. Linn. Soc. 76: 161-176.

Stockey, R. A. 1975: Seeds and embryos of Araucaria mirabilis. - Amer. J. Bot. 62: 856-868.

Stockey, R. A. 1980 : Anatomy and morphology of Araucaria sphaerocarpa Carruthers from the jurassic inferior oolite of Bruton, Somerset. - Bot. Gaz. 141: 116-124.

Stockey, R. A. 1982: The Araucariaceae: an evolutionary perspective. - Rev. Palaeobot. Palynol. 37: 133-154.

Stockey, R. A. 1994: Mesozoic Araucariaceae: Morphology and systematic relationships. - J. Plant Res. 107: 493-502.

Taylor, T. N., Taylor, E. L. \& Krings, M. 2009: Palaeobotany: The biology and evolution of fossil plants, 2nd ed. Burlington.

Wieland, G. R. 1929: The world's two greatest petrified forests. - Science 69: 60-63.

Zonneveld, B. J. M. 2012: Genome sizes of all 19 Araucaria species are correlated with their geographical distribution. Plant Syst. Evol. 298: 1249-1255.

\section{Internetseite}

https://minerando.de/fossilien/versteinerte-hoelzer/araukarienzapfen/

\section{Anschrift des Autors und der Autorin}

Dr. Veit M. Dörken, Universität Konstanz, Fachbereich Biologie M 613, Universitätsstraße 10, 78457 Konstanz, E-Mail: veit.doerken@uni-konstanz.de

Dr. Hilke Steinecke, Palmengarten Frankfurt, Siesmayerstr. 61, 60323 Frankfurt, E-Mail: hilke.steinecke@stadt-frankfurt.de 